\title{
Natural ventilation as a key Airborne Infection Control Measure for Tuberculosis Care Facilities.
}

\author{
Ar. Raja Singh (Corresponding Author), Research Scholar, Department of Architecture, \\ School of Planning and Architecture, New Delhi.
}

A review article.

Tuberculosis or TB has a large number of cases in India. India is fighting TB with the aim of eliminating it by 2025 (1) (2). TB, an airborne disease, has a risk of being hospital acquired (nosocomial) by the patients, visitors and the healthcare workers (HCWs). As a fact, till 2004, there were no studies on nosocomial Tuberculosis in India. (1) (3) The Guidelines on AIC or Airborne Infection Control (4) in Healthcare and other settings, released by the Directorate General of Health Services(Government of India) highlights the role of architects and engineers in Health Infrastructure Design for better infection control. The National Strategic Plan For Tuberculosis Elimination 2017-2025 (2) mentions AIC by highlighting the role of Natural Ventilation in limiting the infection spread in TB care facilities. Like TB, there are other diseases which also spread through the airborne route like Legionella pneomophila, Serratia marcescens and SARS (5). These are also linked to the ventilation of the hospital and have at multiple times revived the interest in the area of Indoor Air Quality for Healthcare facilities though there is an equal risk in Prisons, hostels, homeless facilities and long term care facilities. It was confirmed by the SARS epidemic in 2003 that the 'mechanisms of respiratory disease transmission are still poorly understood.' (6) and, there is a 'lack of scientific evidence underpinning minimum ventilation rate guidelines (5).' The Indian government literature too, mentioned above doesn't have quantitative ventilation guidelines. The AIC guidelines have three types of measures namely, Administrative, Personal Protective and engineering/environmental control (7). The cost of many suggested engineering measures are high, and the more affordable 
measures include improving natural ventilation by opening the windows (8). Ventilation systems not only have an issue with air changes but also are likely to be compromised by poor design, faulty construction or inadequate maintenance which has led to the occurrences of TB (and related diseases) outbreaks. (8)There have been studies in Thailand and Peru which state that naturally ventilated rooms had a higher air change rate. (8) (9)The study in Peru also went on to state that the highest risk of infection occurred in mechanically ventilated rooms with sealed windows, despite being ventilated at recommended rates. As the thrust towards energy-efficient buildings increases, there is a higher chance of making compact spaces which are airtight and use HVAC. (10)Such buildings have been studied to report an increasing trend of airborne infections. There is a need to consolidate the literature available and perform further studies to provide functional and quantitative guidelines to architects for better AIC. (1) Architects, and other infrastructure professionals, need to play a proactive role driven by performance-based research and evidence-based design to design better TB care facilities with the aim of reducing the airborne infection. For this, there needs to be a special focus on the use of natural ventilation and its unexplored potential for infection control.

\section{Keywords: Airborne Infection Control; Natural Ventilation; Nosocomial Tuberculosis; Healthcare Architecture;}

\section{Introduction:}

There have been an estimated 2.8 million new Tuberculosis cases in the world. India accounts for $1 / 4^{\text {th }}$ of these cases. (1) This disease is spread through the airborne route and it is very important to prevent the infection using Airborne Infection Control measures. Healthcare-associated outbreaks in many parts of the world have warranted attention for Tuberculosis infection controls especially in HIV and TB care facilities which go hand in hand as HIV patients are immunosuppressed and are most likely to catch the infection, especially when the possibility of getting the infection from the community is high in the developing countries. This possibility of getting infected is even higher when a person goes to a TB care facility. Healthcare workers are at the greatest risk of getting 
nosocomial infection from their workplace. Along with TB, there have been other diseases which have been linked to airborne infection spread. (5) This had led to an active interest of researchers worldwide in Airborne Infection Control. This Airborne infection control is achieved through Administrative measures, Engineering/Environmental measures and Personal Protective measures (7). The scope of the architects and other healthcare infrastructure professionals is limited to the Engineering/Environmental Control measures. This article will portray Airborne Infection Control as an Indoor Air Quality issue linked to Natural ventilation (8) as a trained architect would perceive it.

\section{Architecture Design for Healthcare}

Hospital Design composes of the indoor environment, Interior Design and the configuration. The configuration is the design of the relative locations and the adjacencies of the spaces. Interior Design is about the furniture, fixtures and the materials used. The indoor environment in a hospital deals with noise, lighting, psychological comfort, the air quality, etc. (11) According to the National Airborne Infection Control Guidelines, architects play a key role in the Health Infrastructure Design. It highlights their role in the reduction of nosocomial infection not only by the means of optimized patient flow patterns but also by finding other affordable solutions to improve the organization of existing and new facilities. This opens us the area of evidence-based design which evaluates the design of healthcare facilities by relying on post-construction design flaws. This can be done by mock-up studies or by simulations. (12) Evidence-based design prescribes design decisions to be based on strong research footing. Testing of proposed architectural designs before construction allows for the improvement of designs leading to the development of safer hospitals. (12) An analysis by an army hospital administrator stated that 'most hospitals in the developing countries are not scientifically designed' He not only mentioned about the configuration issues but also highly a key issue of the required air conditioning and ventilation in hospitals. He mentioned that these requirements, not being properly met, increase the risk of infection transmission. (13)With respect to the modern practice of designing healthcare buildings, some researchers believe that the inclination towards providing comfort to the users favours pathological persistence. (5) TB has made recent 
resurges in New York, USA (14). This is an example of a developed country where Nosocomial infections are among the leading causes of death (15)and there the health benefits of well-planned architecture have been recognized.

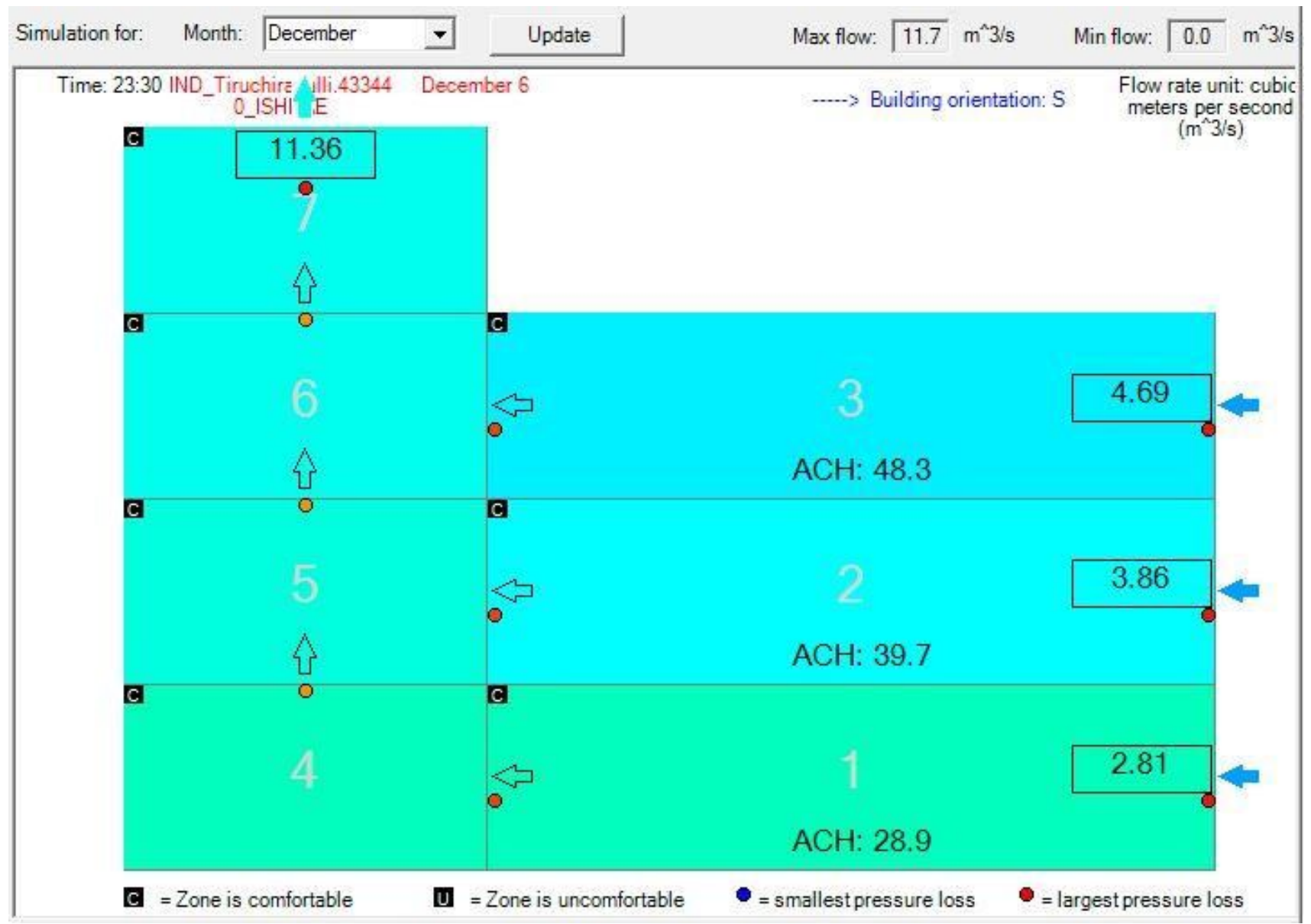

Figure 1: Simple simulation for Natural Ventilation done on MIT Coolvent software can provide a guideline for architects for Natural Ventilation Design (Picture Source: Ar. Raja Singh)

\section{Energy Efficient Buildings and Nosocomial TB.}

Natural Ventilation was believed to be effective in the disinfection of air (5) and TB patients in the past were resorted to a sanatorium. (16) These had a serene environment with stress on natural ventilation and sunlight. With time, there was an improvement of anti-infective strategies and improvement in living conditions. (5) That led to less attention being paid to ventilation as an airborne infection control tool. (5) The aim was shifted to create indoor spaces which had more comfort and which would remove odours. The priority given to comfort was later challenged due to the 1973 Oil Embargo which led to an energy crisis. Now comfort provided has to cost-effective and hence, energy-efficient which was possible by using recirculated air and reducing air change rates. 
Buildings were made tighter and were sealed to trap thermal energy in the spaces. The highly researched 'sick building syndrome' (17) was most likely an outcome of these practices mentioned. Studies have also linked increased patient risk to building smaller rooms which are cheaper to build and are air-conditioned, leading to overcrowding and 'becoming stuffy' leading to an upward trend of infection caused by airborne micro-organisms. (8) People may argue that an energy-efficient building with a properly designed HVAC system may be good enough to dilute the concentration of micro-organisms, but the study done in Peru contradicts this and goes on to state that 'the highest risk of infection occurred in mechanically ventilated rooms with sealed windows, despite being ventilated at recommended rates' (8) Simulation techniques (Figure 1) are useful in the design process to ascertain the probable natural ventilation possible in the spaces.

\section{Infection Spread due to Mechanical Ventilation.}

Ventilation Systems in hospitals have a likeliness of being ill-designed, poorly constructed and not being maintained properly. And 'these failings have been implicated in outbreaks of tuberculosis.' (5) To give an example of other diseases, the source of Srratia marcescens outbreak in a UAE baby hospital was tracked to the duct of an air conditioning system. (18) Faulty and/or contaminated hospital ventilation systems have also been linked with the outbreaks of MRSA. In 1991, Operation Desert Shield, there was a higher infection (respiratory tract) in military personnel in air-conditioned barracks than those in tents. (19) Another study links mechanically ventilated patient rooms to the presence of 'an ecologically distinct set of microbial taxa than those found in the outdoor air.' This was due to the low Phylogenetical Diversity of the bacteria found indoors and they were associated with the clades that were commonly associated with humans as pathogens. The fact that there has been a reduction in the contact with the outdoor environment to make the building more sterile actually may actually do its opposite. This isolation of the indoor environment may not be the most optimal design strategy for bacterial pathogen management. This has led to a rekindling of our interest in the natural disinfection characteristics of outdoor air which were ignored in the past. (15) 


\section{Natural Ventilation and its role in Airborne Infection Control.}

In the context of this paper, natural ventilation means buoyancy and diffusion-driven movement of air. This should not be made synonymous to speedy air movement due to windows which are open or closed and dependent only upon the velocity of the air. In studies, the diffusion of air took even when

there was no wind and the window was partly closed. (8) It has been shown that high levels of protective natural ventilation are readily available and achievable even at low wind speeds. (8)

In 1894, exposure to fresh air 'appeared to reduce the virulence of the tubercule bacillus.' The bacteria were retaining their 'infectivity' in the absence of fresh air. During 1918 influenza pandemic, patients accommodated in the open-air survived in greater number than those kept in the hospital wards. (5)A keystone study was performed by researchers in Lima, Peru in 5 Hospital's 70 rooms in which they conducted 368 experiments. They established that the naturally ventilated rooms (due to open windows) which were made pre-1950s and the ones made during the times of 'modernism' in the 1980s were way more efficient in reducing the risk of tuberculosis transmission than the rooms of the hospital developed in the year 2000 with mechanical ventilation and smaller (tighter) rooms. For all naturally ventilated facilities, opening windows and doors provided median absolute ventilation of 2,477 $\mathrm{m} 3 / \mathrm{h}$, more than six times the $402 \mathrm{~m} 3 / \mathrm{h}$ calculated for mechanically ventilated rooms at $12 \mathrm{ACH}(8)$

The fact that naturally ventilated wards can achieve higher air change rates has been established by many more studies. Case studies from China show that cross ventilation is useful for controlling another airborne infection (SARS). When the proportion of operable windows increased in the isolation ward, there was a better result in preventing outbreaks of SARS among healthcare workers. (20) A study performed in Thailand also reaffirmed that air change rates in naturally ventilated hospital rooms were mostly higher than recommended standards. Ventilation rates were found out to be inadequate in work areas having air conditioning and they found that these were the areas with the highest nosocomial tuberculosis infection risk namely the radiology and emergency departments. (9) 
Natural Ventilation is best suited for the limited resource settings and tropical climates, which also coincidentally have the highest institutional TB transmission risk and the maximum TB burden. In India, the hospitals, especially the public-owned facilities have reported to being crowded and poorly ventilated. (1) In such cases and elsewhere, Ventilation remains a key design strategy to mitigate the spread of infectious airborne diseases indoors. (8) The rates and determinants of natural ventilation in healthcare facilities have not been defined and are an area of continuous research. (8) From the Indian Context, it opens up lots of possibilities for an interdisciplinary study driven by people from healthcare, architecture and facilities management.

\section{Role of microbes for safety: The counterintuitive study}

Microbes are naturally found everywhere. As seen earlier, selected studies on Natural Ventilation and its impact on Nosocomial TB reduction have been performed. In another such study, it has been hypothesized that 'filtration by mechanical ventilation is a form of dispersal limitation, resulting in indoor microbial communities that represent a subset of outdoor microbes.' However, indoor environments have been found to harbour microbial taxa not commonly found outdoors. These indoor taxa have a common resemblance with human-related DNA and hence they have a likeliness to be from the same subfamily of human pathogens. Hence this study talks not about eliminating bacteria, but in selecting the right bacteria. This opens up the area of architectural probiotics. There is a scope in the exploration of the "good" bacteria that architects can select for their indoor environments. (15) This area requires further study w.r.t. the tuberculosis bacteria and its position in these dynamic spatial system composed of bacteria and other micro-organisms.

\section{Disadvantages of relying on Natural Ventilation with possible solutions.}

To provide a balanced view it is important to discuss the possible disadvantages of Natural

Ventilation for a Tuberculosis facility. In places with extreme climates, both hot and cold, sometimes opening up windows for natural ventilation is likely to cause thermal discomfort to the inhabitants.

Hence a balance has to be established between thermal comfort, air movement and ventilation for the 
dilution of the bacterial concentration. A solution to this can be decentralized cooling units in rooms which have operable windows for intentional ventilation whenever required. Non-operable windows should be avoided in almost all cases.

In some cases, windows have shown to be non-operable due to maintenance issues. There may be administrative reasons (like the absence of window opening/closing staff). Sometimes due to poor organization of spaces windows are concealed by the placement of furniture or closets. In these cases, the design, as intended may not perform.

Other issues may be the increased quantity of pollen, particulate matter and gaseous pollutants that may enter through the window openings. (5) This can be tackled with the use of innovative filters that can be used in the window assemblies. (21)

\section{Methodology of performing studies}

Study in India involved a baseline assessment (7)of 35 health centres across three Indian states- West Bengal, Gujrat and Andhra Pradesh. It was a very basic risk assessment methodology which involved basic equipment to measure ventilation and air change rates. The equipment was: 1 . Incense sticks 2 . Measuring tape for room volume measurement 3. Swing Vane Anemometer 4. Air anemometer and 5. digital cameras. This can be appreciated as an Indian study which has an empirical methodology, but it has certain limitations. It did not perform any surveillance tests on the human subjects, or TB bacterial counts and analysis. It also did not have a $\mathrm{CO} 2$ dilution tracer gas test to determine the air changes per hour more accurately.

Another study by Escombe et al in Lima, Peru used the tracer gas method. It also used a mathematical model called the Wells-Riley model to establish a correlation between the infection risk assessment and the air changes per hour derived from the above mentioned $\mathrm{CO} 2$ tracer gas technique. It has very effectively given us one of the easiest methods to compare between two use cases of natural ventilation. The comparison between a mechanical ventilated and a naturally ventilated case has also been demonstrated. Combined with the Wells Riley Model, it provides an 
indication without resorting to actual bacteria samplings, culturing, identification and counting routine. The issue highlighted by some researchers is that Wells Riley is a static model and it may not be an actual indicator of infection caused to Humans. The study also does not talk about the proximity of the person to the infection source and it considers the whole space to be homogeneous for this purpose. (8)

Another study performed by researchers in Pakistan by Asif et al, took samples of micro-organisms using air samplers, followed by the culturing of bacteria and fungi which were then identified and counted. This was done using microscopic analysis at $1000 \mathrm{x}$ magnification, followed by oil immersion and gram staining. The identification was done using Bergey's Manual of determinative bacteriology. The counting was done in the unit CFU/m3. Statistical analysis was followed. This gave a quantitative idea of bacteria and fungi population present in the air. The study concluded that the microorganisms were more where the occupancy was more. The study did not compare natural ventilation with a mechanical ventilation case. There was also no study on the air changes in the spaces where the sampling was done. (10)

In another study by Kembel et al., three samples were collected. One was from a mechanically ventilated (duct supplied) room in a hospital. The other was a room where the windows were made operable and were opened with the air conditioning switched off. The third was the outside environment used as a control case. Samples were collected from each of the three cases followed by bacterial cell density estimation, DNA extraction and bacteria 16S gene amplification. This was done in order to very accurately identify the bacteria and fungi for a taxonomically accurate analysis. The goal of which was to find out the Phylogenetic Diversity of the sampled microorganisms in order to classify and compare their populations and diversity in the different zones. (15)

The tracer $\mathrm{CO} 2$ gas dilution analysis method is a suitable method to find the relative rates of diffusion in different spaces. These rates of diffusion provide an idea of the Air changes which can be further substituted in the Wells Riley model to check for infection transmission risk. This may 
seem like a proxy test which can be made more valid by actually performing a sampling of bacteria from the air followed by identifying and counting them.

There is also immense scope in utilizing Computational fluid dynamics based simulation modelling software to analyse the movement of air and pathogenic bacteria in the TB care facilities.
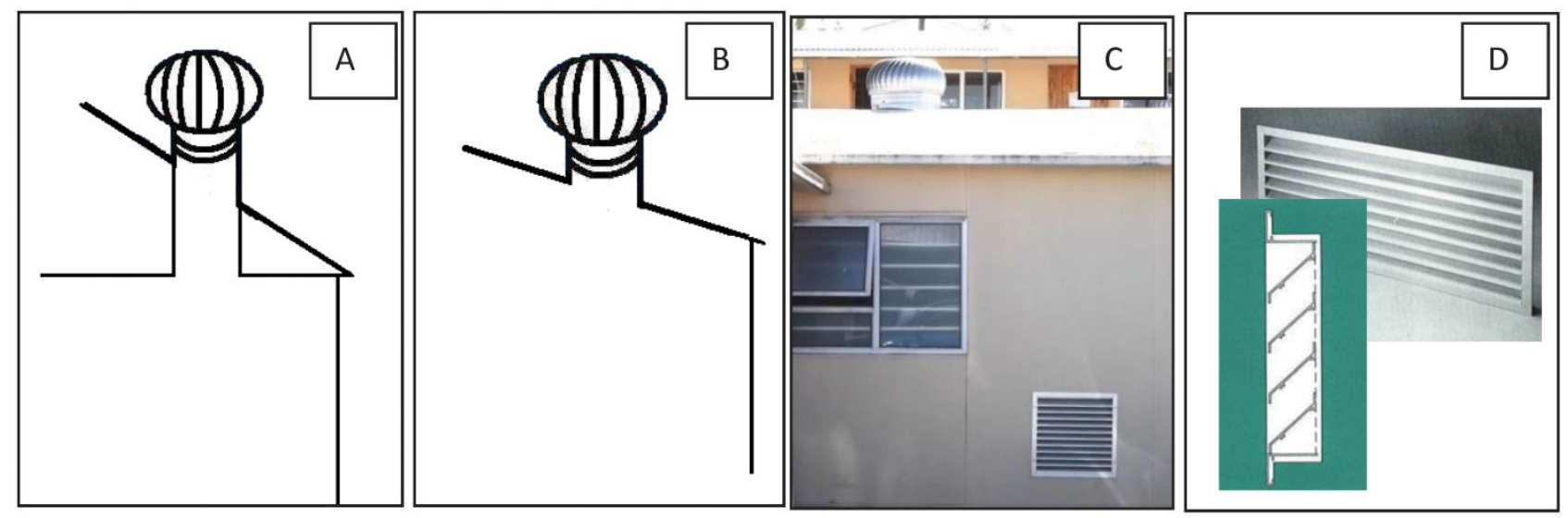

Figure 2: Installation of roof turbines and air-intake grates. A simple method of Reinforced Natural Ventilation: (Picture Source: Wind-Driven Roof Turbines: A Novel Way to Improve Ventilation for TB Infection Control in Health Facilities

Cox H, Escombe R, McDermid C, Mtshemla Y, Spelman T, et al. (2012) Wind-Driven Roof Turbines: A Novel Way to Improve Ventilation for TB Infection Control in Health Facilities. PLOS ONE 7(1): e29589.)

\section{The way ahead for Infrastructure designers.}

It has been established that natural ventilation, with some defects, has a certain advantage over mechanical ventilation for reduction of infection transmission risk. There has been a study in South Africa where the planners have used a wind-driven roof turbine system along with inlet air gratings to enhance the ventilation in a TB facility. (22) In places with extreme climates where natural ventilation may not be possible, the use of upper room Ultraviolet has also been researched and advocated. (23) TB is a good starting point (1) as the TB facilities can be used as a model for other diseases caused by airborne infection spread. Addressing the nosocomial infection spread is also particularly helpful for the HIV care centres where the patients are most susceptible to all kinds of airborne infections due to their suppressed immunity. In designing a healthcare facility, an architect has to face many constraints and the choice has to be in consistence with a balanced solution which takes into consideration many factors at once. While providing comfort may be important for a 
commercial building or a hotel, it may be secondary for a Tuberculosis facility where infection control is primary and providing comfort may put infection control into jeopardy. There has been an active shift towards performance in architectural design (24) and the building needs to perform well on the infection control front too. Hybrid systems where decentralized air conditioning may be provided along with operable windows can be a possible solution where further quantitative research may be done. Figure 2 shows one method where a wind-driven turbine was used and tested for Natural Ventilation. Other solutions could be more advanced natural ventilation systems which prohibit the entry of dust, pollen and gaseous pollutants into the indoor spaces. The need to address nosocomial transmission will not only help the patients and visitor to TB facilities but will impact Healthcare workers the most. These measures as a whole will contribute towards widening and deepening the scope of the involvement of infrastructure professionals for the common goal of eradication of TB in India by 2025.

\section{Declaration:}

No funding was received for this study.

\section{Contribution of Authors:}

RS has done the literature analysis and has critically reviewed the papers and presented the work. Email of the Corresponding Author: raja.singh.email@gmail.com

\section{Conflicts of Interest}

The author has none to declare.

\section{Works Cited:}

1. Nosocomial Tuberculosis in India. Pai, Madhukar, et al., et al. 2006, Emerging Infectious Diseases, CDC.

2. Central TB Division, Directorate General of Health Services, MoHFW, Govt. of India. National Strategic Plan for Tuberculosis Elimination 2017-2025. New Delhi. : Central TB Division, Directorate General of Health Services, MoHFW, Govt. of India, 2017.

https://tbcindia.gov.in/WriteReadData/NSP\%20Draft\%2020.02.2017\%201.pdf. 
3. Tuberculosis infection control measures at health care facilities offering HIV and tuberculosis services in India: A baseline assessement. Sachdeva, Kuldeep Singh, et al., et al. 2018, Indian Journal of Tuberculosis, p. 263.

4. Directorate General of Health Services, MoHFW, Govt. of India. Guidelines on Airborne Infection Control in Healthcare and Other Settings: In the context of tuberculosis and other airborne infections. New Delhi : Directorate General of Health Services, MoHFW, Govt. of India., 2010.

5. Roles of sunlight and natural ventilation for controlling infection: historical and current perspectives. Hobday, R A and Dancer, S J. 2013, Journal of Hospital Infection, pp. 271-282.

6. Room ventilation and the risk of of airborne infection transmission in 3 health care settings within a large teaching hospital. Knibbs, L D, et al., et al. 2011, American Journal of Infection Control, pp. 866-872.

7. Airborne infection control in India: Baseline assesement of health facilities. Parmar, Malik M, et al., et al. 2015, Indian Journal of Tuberculosis, pp. 211-217.

8. Natural Ventilation for the Prevention of Airborne Contagion. Escombe, A Roderick, et al., et al. 2007, PLOS Medicine.

9. Inadequate ventilation for nosocomial tuberculosis prevention in public hospitals in Central Thailand. Jiamjarasrangsi, w, et al., et al. 2009, International Journal of Tuberculosis and Lung Diseases, pp. 454-459.

10. Microbial quality assesement of indoor air in a large hospital building during winter and spring seasons.

Asif, Ayesha, et al., et al. 2018, Building and Environment , pp. 68-73.

11. The Architecture of Safety: hospital design. Joseph, Anjali and Rashid, Mahbub. 2007, Current Opinion in Critical Care, pp. 714-719.

12. Patient safety begins with proper planning: a qualitative method to improve hospital design. Birnbach, D J, et al., et al. 2010, QUAL SAF HEALTH CARE, pp. 462-465.

13. Desisgning Hospital for better Infection Control: an Experience. Rao, Lt Col SKM. 2004, MJAFI, pp. 63-66.

14. Characterization of infectious aerosols in healthcare facilities: an aid to effective engineering controls and preventive strategies. Cole, E C and Cook, C E. 1998, American Journal of Infection Control, pp. 453-464.

15. Architectural Design influences the diversity and structure of the built environment microbiome. Kembel, Steven W, et al., et al. 2012, The International Society for Microbial Ecology Journal, pp. 1469-1479.

16. Yuko, Elizabeth. How the Tuberculosis Epidemic Influenced Modernist Architecture. www.citylab.com. 30 October 2018.

17. Building related illness: current concepts. Menzies, D and Bourbeau, J. 1997, N Engl J Med, pp. 1524-1533.

18. An outbreak of Serratia marcescens infection in special-care baby unit of a community hospital in United Arab Emirates: the importance of the air conditioner duct as a nosocomial reservoir. Udaman, S A, Farrukh, A S and Nath, K N. 2002, Journal of Hospital Infections, pp. 175-180.

19. Respiratory disease among military personnel in Saudi Arabia during Operation Desert Shield. Richards, A L, et al., et al. 1993, American Journal of Public Health, pp. 1326-1329. 
20. Investigating a safe ventilation rate for the prevention of indoor SARS transmission: an attempt made on simulation approach. Jiang, Y, et al., et al. 2009, Building Simulation, pp. 281-189.

21. Indian Institute of Technology, New Delhi. Pollution Net | Nasofilters. [Online] https://nasofilters.com/pollution-net/.

22. Wind-Driven Roof Turbines: A Novel Way to Improve Ventilation for TB Infection Control in Health Facilities. Cox, Helen, et al., et al. 2011, PLOS One, p. e29589.

23. Upper-Room Ultraviolet Light and Negative Air lonization to Prevent Tuberculosis Transmission. Escombe, A Roderick, et al., et al. 2009, PLOS Medicine, p. e1000043.

24. Building performance simulation in the early design stage: An introduction to integrated dynamic models. Negendahl, Kristoffer. 2015, Automation in Construction, pp. 39-53.

25. How the Tuberculosis Epidemic Influenced Modernist Architecture. Yuko, Elizabeth. 2018, www.citylab.com.

26. Tuberculosis infection control measures at healthcare facilities offering HIV and tuberculosis services in India: A baseline assesement. Sachdeva, K S, et al., et al. 2018, Indian Journal of Tuberculosis. 